OPEN ACCESS

Edited by:

Xiao Zhu,

Guangdong Medical University, China

Reviewed by:

Fengchao Lang,

University of Pennsylvania,

United States

Chen Li,

Charité-Universitätsmedizin

Berlin, Germany

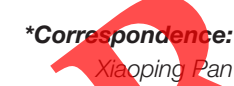

dr_panxp5

Specialty section:

This article was submitted to

Cancer Genetics,

a section of the journal

Frontiers in Oncology

Received: 12 December 2019

Accepted: 12 March 2020

Published: 30 April 2020

Citation:

Pan X, Hong X, Lai J, Cheng L,

Cheng Y, Yao M, Wang R and Hu N (2020) Exosomal MicroRNA-221-3p

Confers Adriamycin Resistance in

Breast Cancer Cells by Targeting PIK3R1. Front. Oncol. 10:441

doi: $10.3389 /$ fonc. 2020.00441

\section{Exosomal MicroRNA-221-3p Confers Adriamycin Resistance in Breast Cancer Cells by Targeting PIK3R1}

\author{
Xiaoping Pan ${ }^{1 *}$, Xiaolv Hong ${ }^{2}$, Jinguo Lai ${ }^{1}$, Lu Cheng ${ }^{1}$, Yandong Cheng ${ }^{3}$, Mingmei Yao ${ }^{1}$, \\ Rong Wang ${ }^{1}$ and $\mathrm{Na} \mathrm{Hu}^{1}$
}

${ }^{1}$ Clinical Laboratory, Huadu Hospital, Southern Medical University, Guangzhou, China, ${ }^{2}$ Department of Infectious Diseases, Huadu Hospital, Southern Medical University, Guangzhou, China, ${ }^{3}$ Department of Breast Surgery, Hurdu Hospital, Southern Medical University, Guangzhou, China

Drug resistance in breast cancer $(\mathrm{BC})$ cells continues to be a stern obstacle hindering $\mathrm{BC}$ treatment. Adriamycin ( $\mathrm{ADR}$ ) is a frequently employed chemotherapy agent used to treat $\mathrm{BC}$. The exosomal transfer of micro RNAs (miRNAs) has been reported to enhance the drug-resistance of $\mathrm{BC}$ cells. Herein, we first sought to elucidate the possible role of the exosomal transfer of niR-221-3p in the drug resistance of MCF-7 cells to ADR. Differentially expressed genes (DEGs) were initially screened through microarray analysis in BC drug resistance-rellated datasets. Next, the expression of miR-221-3p and phosphoinositide-3-kinase regulatory subunit 1 (PIK3R1) was quantified in ADR-resistant MCF-7 (MCF-7/ADR) and ADR-sensitive MCF-7 (MCF-7/S) cell lines, after which exosomes were separated and identified in each cell line. Target relationship between miR-221-3p and PIK3R1 was validated by a dual-luciferase reporter assay. Next, the expression of miR-221-3p and PIK3R1 was altered to clarify their effects on the resistance of MCF-7 cells to ADR in vitro and in vivo. PIK3R1 was identified as a $B C$ drug resistance-related DEG, with the regulatory miR-221-3p subsequently obtained. Moreover, the MCF-7/ADR cells exhibited a low expression of PIK3R1 and a high expression of miR-221-3p. Notably, PIK3R1 was identified as a target gene of miR-221-3p. The overexpression of miR-221-3p in MCF-7/ADR cell-derived exosomes promoted ADR resistance in MCF-7/S cells via the PI3K/AKT signaling pathway. The in vitro results were reproducible in in vivo assays. Taken together, drug-resistant $B C$ cell-derived exosomal miR-221-3p can promote the resistance of BC cells to ADR by targeting PIK3R1 via the PI3K/AKT signaling pathway in vitro and in vivo. These findings provide encouraging insights and provide perspectives for further investigation into the $\mathrm{BC}$ drug resistance mechanism.

Keywords: breast cancer, exosomes, microRNA-221-3p, phosphoinositide-3-kinase regulatory subunit 1, drug resistance, adriamycin

\section{INTRODUCTION}

Breast cancer (BC) remains one of the most common and deadly malignancies afflicting the female population (1). The incidence of the young population with $\mathrm{BC}$ is particularly higher in China in comparison to the western world (2). Several treatment agents exist for the treatment of BC, all with varying efficacies. Adriamycin (ADR) has been well-documented to play a crucial role in treating 
BC, owing to its ability to kill cancer cells in humans (3). However, the emergence of drug resistance remains a complex issue that can accelerate drug efflux or even alter an individual's immune system, highlighting a significant obstacle regarding the treatment of patients with cancer (4). A previous report has defined BC drug resistance as a clinical condition implicated by molecular variations (5).

Investigations into the underlying molecular mechanisms associated with drug resistance continue to be conducted. Exosome transfer has been highlighted as a potent medium capable of elucidating the mechanisms associated with drug resistance (6). Exosomes can be found in human body fluids, and their anti-cancer effects, by transferring drugs outside of BC cells, have been proven in a previous study, resulting in drug resistance (7). Accumulating evidence has emphasized the significance of exosomal microRNAs (miRNAs) in drug resistance. At present, 22 exosomal miRNAs have been identified in BC cell resistance, whose altered expression has been suggested to be indicative of prognostic or diagnostic markers for drug resistance in BC (8). Although the role of exosomal miRNAs in $\mathrm{BC}$ drug resistance has been noted, the specific mechanisms by which exosomal miRNAs contribute to drug resistance remain largely unknown and thus require further investigation. The upregulation of miR-221-3p has been implicated in cervical cancer, whereby cancer-derived exosomal miR-221-3p was found to significantly promote tumor growth (9). Moreover, oncogene-miR-221 has also been reported to play a role in the mechanism of drug resistance in non-small cell lung cancer (10). Although miR221-3p has been extensively investigated in BC (11-14), the specific function of BC-secreted exosomal miR-221-3p in BC drug resistance still remains unclear. A recent study explored the role of miR-221 targeting phosphoinositide-3-kinase regulatory subunit 1 (PIK3R1) in cholangiocarcinoma cells (15). The forced expression of PIK3R1 possesses the ability to significantly enhance paclitaxel resistance in BC cells and xenograft tumors and enhanced PIK3R1 expression also leads to activated phosphatidylinositol 3-kinase/protein kinase B (PI3K/AKT) (16). Based on the aforementioned exploration of the literature, we hypothesized that an exosome-mediated transfer of miR-221-3p in ADR-resistant BC cells may be involved in $\mathrm{BC}$ drug resistance, by regulating PIK3R 1 and the PI3K/AKT signaling pathway. The central objective of the study was, therefore, to determine if the aforementioned hypothesis was valid and to further explore the mechanisms by which exosomal miR-221-3p promoted the resistance of $\mathrm{BC}$ cells to $\mathrm{ADR}$ through the regulation of the PIK3R1-dependent PI3K/AKT signaling pathway.

\section{MATERIALS AND METHODS}

\section{Ethics Statement}

The study was approved by the Animal Ethics Committee of Huadu Hospital, Southern Medical University. All animal experiments were performed in strict accordance with the Guide for the Care and Use of Laboratory Animals published by the US National Institutes of Health. Extensive efforts were made to minimize the number and suffering of the included animals.

\section{Microarray-Based Gene Expression Profiling}

A BC-resistant microarray dataset GSE76540 including BC cell lines ADR-sensitive (MCF-7/S) and ADR-resistant (MCF7/S/ADR) was obtained from the GEO database (https://www. ncbi.nlm.nih.gov/geo/). The sequencing platform was GPL570 [HG-U133_Plus_2] Affymetrix Human Genome U133 Plus 2.0 Array. Next, differential analysis on gene expression in the two cell lines was conducted using the limma microarray package, with the differentially expressed genes (DEGs) subsequently screened out using the threshold of $\mid$ log Fold Change $\mid>$ 2 and $p$-value $<0.05$. A protein-protein interaction (PPI) map on DEGs was then plotted using the STRING database (https://string-db.org). Next, miRNA expression in BC exosomes was determined using the EVmiRNA database (http://bioinfo. life.hust.edu.cn/EVmiRNA\#!/browse). The upstream regulatory miRNA of PIK3R1 was predicted by the mirDIP (http://ophid. utoronto.ca/mirDIP/index.jsp\#r) and TargetScan (http://www. targetscan.org/vert_71/) databases, after which the binding site between miRNA and mRNA was detected.

\section{Cell Culture and Treatment}

Human normal breast epithelial cell line MCF-10A, and human BC epithelial cell line MCF-7/S and MCF-7/ADR purchased from the Cell Bank of Typical Culture Preservation Committee of Chinese Academy of Science (Shanghai, China) were cultured with Roswell Park Memorial Institute (RPMI)-1640 medium containing 10\% fetal bovine serum (FBS) and 10\% penicillinstreptomycin (Gibco Life Technologies, Grand Island, NY, USA) in a $5 \% \mathrm{CO}_{2}$ incubator at $37^{\circ} \mathrm{C}$. The cells were then detached using $0.25 \%$ trypsin, and subsequently sub-cultured at a ratio of 1:3. Next, the cells were seeded into a six-well-plate at a density of $\times 10^{5}$ cells/well. When reaching $70-80 \%$ confluence, the cells at logarithmic growth phase were selected for further experiments.

MCF-7/S and MCF-7/ADR cell lines were seeded into sixwell-plates at a density of $4 \times 10^{5}$ cells/well and then transfected in accordance with the protocol provided by lipofectamine 2000 (11668-019; Invitrogen, Carlsbad, CA, USA). A total of $10 \mu \mathrm{g}$ diluted plasmids (a final concentration of $50 \mathrm{nM}$ ) by $250 \mu \mathrm{L}$ serum-free Opti-MEM and $5 \mu \mathrm{L}$ diluted lipofectamine 2000 by $250 \mu \mathrm{L}$ serum-free Opti-MEM were mixed, allowed to stand for $20 \mathrm{~min}$, and added into the six-well-plate, followed by $48 \mathrm{~h}$ culturing at $37^{\circ} \mathrm{C}$ in $5 \% \mathrm{CO}_{2}$ and saturated humidity. Next, the cells were then further cultured for $24-48 \mathrm{~h}$ with RPMI1640 medium containing 10\% FBS for subsequent analyses. The MCF-7/S cells were transfected with plasmids of overexpression negative control (NC) (oe-NC), oe-PIK3R1, short hairpin RNA (shRNA) against NC (sh-NC), and sh-PIK3R1. MCF-7/ADR cells were transfected with plasmids of mimic NC, miR-221-3p mimic, inhibitor NC, miR-221-3p inhibitor, oe-NC, oe-PIK3R1, sh-NC, and sh-PIK3R1. The aforementioned sequences and plasmids were all purchased from Shanghai GenePharma Co., Ltd. (Shanghai, China).

\section{Isolation and Identification of Exosomes}

The MCF-7/S and MCF-7/ADR cells were cultured overnight in serum-free RPMI-1640 medium, followed by the collection 
of supernatant after $24 \mathrm{~h}$. Cell debris were removed by centrifugation at $2,000 \mathrm{~g}$ and $4^{\circ} \mathrm{C}$ for $20 \mathrm{~min}$. The supernatant was harvested and subsequently centrifuged at $10,000 \mathrm{~g}$ and $4^{\circ} \mathrm{C}$ for $1 \mathrm{~h}$. The cells were next suspended and precipitated using the RPMI-1640 medium [treated as the way described in a previous study (17)] with the supernatant, then discarded in accordance with the same procedure stated above. The precipitated cells were stored at $-80^{\circ} \mathrm{C}$ for subsequent use.

The exosomes were identified using a transmission electron microscope (TEM). Briefly, $30 \mu \mathrm{L}$ exosomes were added onto the grid, supplemented with $30 \mu \mathrm{L}$ phosphotungstic acid solution $(\mathrm{pH}=6.8)$, counterstained at room temperature for $5 \mathrm{~min}$, dried and photographed under a TEM.

The content of CD63, an exosome surface marker, was measured by flow cytometry. The exosomes were first extracted, resuspended in $1 \mathrm{~mL}$ phosphate buffer saline (PBS) with $1 \%$ bovine serum albumin (BSA) and cultured at room temperature for $30 \mathrm{~min}$ to block non-specific antigen. Exosomes were then resuspended in a $200 \mu \mathrm{L}$ eppendorf (EP) tube and supplemented with the CD63-PE antibody, respectively. The tubes free of antibody addition were regarded as blanks, while tubes with PE conjugated anti-human immunoglobulin $\mathrm{G}(\mathrm{IgG})$ were regarded as the isotype control. The cells were centrifuged followed by the removal of the supernatant. The exosomes were resuspended again, loaded, and determined using the Guava easyCyte ${ }^{\mathrm{TM}}$ flow cytometry based on the provided instructions. The expression of the exosome surface markers CD63 and HSP70 was evaluated by western blot analysis, while the level of miR-221-3p contained in the exosome was detected by reverse transcription quantitationpolymerase chain reaction (RT-qPCR).

The diameter of exosomes was evaluated using dynamic light scattering. The evaluation was performed at an excitation wavelength of $532 \mathrm{~nm}$ based on the instructions provided by Zetasizer Nano-ZS90 (Malvern Corporate, Malvern, UK). Th exosome sample was diluted by $0.15 \mathrm{M} \mathrm{NaCl}(1: 50)$ and deteeted by optical signal.

\section{Endocytosis of MCF-7/ADR Cell-Derived Exosomes by MCF-7/S Cells}

The extracted MCF-7/ADR exosomes were labeled by a PKH67 (Green) staining solution (MINI67-1KT, Sigma-Aldrich, St. Louis, MO, USA) according to the provided instructions. The fluorescence-labeled exosomes were then co-cultured with confluent BC cells that had been seeded into 24-well-plates for $48 \mathrm{~h}$ of culturing and observed under an inverted fluorescence microscope. The expression of miR-221-3p and PIK3R1 was then detected by RT-qPCR, with the protein expression of PIK3R1 determined by western blot analysis.

\section{RNA Isolation and Quantification}

Total RNA was extracted by TRIzol reagent (15596-018; Beijing Science \& Technology Co., Ltd., Beijing, China). All primers in this study were synthesized by Takara (Dalian, China; Table 1). Reverse transcription was then conducted following the instructions of the complementary DNA reverse transcription kit (K1622; Beijing Reanta Biotech Co., Ltd., Beijing, China). The relative transcription level of target genes was calculated
TABLE 1 | Primer sequences for RT-qPCR

\begin{tabular}{lll}
\hline Genes & Primer sequences & \\
\hline PIK3R1 & F: 5'-GCTTGCCGAGCCCTATA & R: 5'-ACATTGAGGGAGTCGTT \\
& A-3' & GTG-3' \\
miR-221- & F: 5'-AGTTGAGAAACAAGGGCTC & R: 5'-GTATCCAGTGCAGGGTC \\
$3 p$ & AA-3' & C-3' \\
U6 & F: 5'-CTCGCTTCGGCAGCACA-3' & R: 5'-AACGCTTCACGAATTTG \\
& & CGT-3' \\
$\beta$-actin & F: 5'-GCCGGGACCTGACTGACTA & R: 5'-TTCTCCTTAATGTCACG \\
& C-3' & CACGAT-3'
\end{tabular}

$R T-q P C R$, reverse transcription quantitative polymerase chain reaction; PIK3R1, phosphoinositide-3-kinase regulatory subunit 1; miR-221-3p, microRNA-221-3p; $F$, forward; $R$, reverse.

based on the $2^{-\Delta \Delta C t}$ method, with U6 and $\beta$-actin serving as the loading controls. Cell quantification was performed using the same method.

\section{Western Blot Analysis}

Total protein was extracted using radio-immunoprecipitation assay (RIPA) lysis buffer (Beyotime Biotechnology, Shanghai, China), and protein concentration was measured using the bicinchoninic acid kit (20201ES76; Yeasen Biotech Co., Ltd., Shanghai, China). The protein was then quantified based on different concentrations, separated using polyacrylamide gel electrophoresis, and transferred onto a polyvinylidene fluoride membrane (IPVH85R; Millipore Inc, Bedford, MA, USA). The membrane was subsequently blocked with 5\% BSA at room temperature for $1 \mathrm{~h}$ and then incubated overnight at $4^{\circ} \mathrm{C}$ with the diluted primary rabbit anti-human antibodies against PIK3R1 (1:2,000; ab191606; Abcam Inc., Cambridge, UK), phosphorylated PI3K (p-PI3K) (1:500; BS4605; Bioworld Technology, Inc., NY, USA), PI3K (1:1,000; ab32089; Abcam Inc., Cambridge, UK), phosphorylated AKT (p-AKT) (1:500; ab38449; Abcam Inc., Cambridge, UK), AKT (1:10,000; ab179463; Abcam Inc., Cambridge, UK), and $\beta$-actin (1:5,000; ab8226; Abcam Inc., Cambridge, UK). After three washes with Tris-buffered saline Tween-20 (5 min each), the membrane was supplemented with horseradish peroxidase-labeled goat anti-rabbit IgG (1:20,000; ab205718; Abcam Inc., Cambridge, UK) and developed using a developing solution. The protein was then quantified using the ImageJ 1.48u software (National Institutes of Health, Bethesda, $\mathrm{MD}$, USA). The relative protein expression was presented by the gray value of the target band to that of $\beta$-actin.

\section{3-(4,5-Dimethylthiazol-2-yl)-2, 5-Diphenyltetrazolium Bromide (MTT) Assay}

A total of $200 \mu \mathrm{L}$ cell suspension was added into a 96-well-plate at a density of $5 \times 10^{5}$ cells/well, followed by the addition of $500 \mathrm{ng} / \mathrm{mL}$ ADR $(20 \mu \mathrm{L} \mathrm{ADR} /$ well $)$ with four repeated wells for each concentration, and zero-setting and blank control wells set. The well without the addition of ADR was regarded as blank. The cells were then incubated on days $1-5$ in a $5 \% \mathrm{CO}_{2}$ incubator at $37^{\circ} \mathrm{C}$ and reacted with $\mathrm{ADR}$ at different concentrations. After the 
removal of the medium, $90 \mu \mathrm{L}$ serum-free RPMI-1640 medium and $20 \mu \mathrm{L}$ MTT solution (5 $\mathrm{mg} / \mathrm{mL} ; 0.5 \% \mathrm{MTT}$ ) was added to each well and incubated for $4 \mathrm{~h}$ in a $5 \% \mathrm{CO}_{2}$ incubator at $37^{\circ} \mathrm{C}$. After the incubation was terminated, $200 \mu \mathrm{L}$ dimethyl sulfoxide was added to each well and shaken for $10 \mathrm{~min}$ to repeatedly dissolve the crystals. The cell survival curve was drawn after measuring the optical density at $570 \mathrm{~nm}\left(\mathrm{OD}_{570}\right)$ value using an automatic microplate reader with the cell survival rate (\%) calculated $\left(\mathrm{OD}_{570}\right.$ in the experimental group/OD 570 in the control group $\times 100 \%)$.

\section{Flow Cytometry}

Cell apoptosis was detected using an Annexin V-fluorescein isothiocyanate (FITC)/propidium iodide (PI) apoptosis detection kit (C1062M, Shanghai Beyotime Biotechnology Co., Ltd., Shanghai, China). Briefly, $1 \times 10^{6}$ cells $/ \mathrm{mL}$ were washed twice with cold PBS, then resuspended following the addition of 195 $\mu \mathrm{L}$ Annexin V-FITC and incubated with $5 \mu \mathrm{L}$ Annexin VFITC and $10 \mu \mathrm{L}$ PI under conditions void of light at room temperature for $15 \mathrm{~min}$. Finally, cell apoptosis was quantified by FACSca flow cytometry (FACSVerse/Calibur/AriaIISORP, BD Biosciences, San Jose, CA, USA).

\section{Dual-Luciferase Reporter Assay}

The target relationship between PIK3R1 and miR-221-3p was verified by dual-luciferase reporter assay. The target and mutant sequences were designed based on the binding sequence of PIK3R1 3'untranslated region (UTR) and miR-221-3p. The luciferase activity of PIK3R1 $3^{\prime}$ UTR in cells transfected with miR-221-3p was determined in accordance with the instructions provided by the Genecopoeia's dual-luciferase reporter assay kit (D0010; Beijing Solarbio Science \& Technology Co., Ltd., Beijing, China). Luciferase intensity was then evaluated by Promegas GLomax20/20 Luminometer.

\section{Tumor Xenografts in Nude Mice}

A total of $30 \mathrm{BALB} / \mathrm{c}$ nude mice (aged 3-5 weeks; weighing 14$18 \mathrm{~g}$ ) were housed in a specific pathogen-free barrier facility with regular ultraviolet radiation, sterilized cages, bedding materials, food, and water under controlled temperature and humidity conditions $\left(24-26^{\circ} \mathrm{C} ; 40-60 \%\right)$. The cultured MCF-7 cells were selected, and its suspension concentration was adjusted to $1 \times$ $10^{6}$ cells/mL using PBS. Next, $50 \mu \mathrm{L}$ cell suspension was extracted and subcutaneously inoculated into the right flank of each mouse. One week later, the mice were randomly treated (six mice in each group) with (1) PBS, (2) ADR (25 mg/kg), (3) MCF-7/ADR exosomes added ADR, (4) MCF-7/ADR exosomes with inhibitor NC, and ADR and (5) MCF-7/ADR exosomes with miR-221-3p inhibitor and ADR. After 30 days had elapsed, the mice were euthanized after anesthesia by injections of pentobarbital sodium (100 mg/kg; P3761; Sigma-Aldrich, St. Louis, MO, USA). Their tumors were then isolated and dissected. Tumor volume was then calculated using the formula: volume $=\pi$ (short diameter ${ }^{2} \times$ long diameter)/6. The weight of tumors was finally measured using a balance.

\section{Statistical Analysis}

All data were processed with the SPSS 21.0 statistical software (IBM Corp., Armonk, NY, USA). Measurement data were expressed as mean \pm standard deviation. Unpaired data in compliance with normal distribution and homogeneity of variance between two groups were compared using an unpaired $t$-test. Comparisons among multiple groups were conducted by one-way analysis of variance (ANOVA) with Dunnett's post-hoc test. Data at different time points and different concentrations were compared by repeated measures ANOVA with Bonferroni post-hoc test. A value of $p<0.05$ indicated significant difference.

\section{RESULTS}

\section{PIK3R1 Was Poorly Expressed in Drug-Resistant BC Cells}

The BC drug resistance-related microarray GSE76540 was obtained from the GEO database, including the cell lines MCF7/S and MCF-7/ADR. A total of 2745 DEGs were obtained through differential analyses on gene expression in the two cell lines (Figure 1A). The relationship between the DEGs was analyzed by PPI (Figures 1B,C), the results of which revealed five genes (UBB, GIGT1, PIK3R1, GNB2, and ESR1) located at the center of PPI network. Differential expression analysis of these five genes was subsequently conducted in order to identify their expression in normal breast epithelial cell MCF-10A and ADRsensitive BC cell line MCF-7/S, which displayed that PIK3R1 was the gene with the most variation (Figure 1D). Next, to further termine the expression of PIK3R1 in drug-resistant BC cells, PIK3R1 expression in normal MCF-10A, ADR-sensitive MCF7/S and ADR-resistant MCF-7/ADR cell lines was evaluated RT-qPCR and western blot analysis. The results obtained demonstrated that PIK3R1 was downregulated in MCF-7/ADR cells in contrast to that in MCF-10A and MCF-7/S cells $(p<$ 0.05; Figures 1E,F). Altogether, the results obtained indicated that PIK3R1 was involved in BC drug resistance.

\section{PIK3R1 Regulated Cell Apoptosis and Drug-Resistance of BC Cells}

Following the confirmation of PIK3R1 contribution to BC drug resistance, we set out to further investigate the role of PIK3R1 in drug-resistance BC cells. After PIK3R1 was overexpressed or knocked down, the expression of PIK3R1 in the MCF-7/S and MCF-7/ADR cells was determined by RTqPCR and western blot analysis. As depicted in Figures 2A-D, PIK3R1 expression was elevated in cells overexpressing PIK3R1 compared to NC transfection, while the expression of PIK3R1 was decreased in sh-PIK3R1-transfected cells in contrast to sh-NC-transfected cells (all $p<0.05$ ). The differentlytreated cells were then processed with ADR with various concentration, among which the MCF-7/S cells were treated with 0.03/0.1/0.3/1/3/10/30 $\mu \mathrm{g} / \mathrm{L}$ ADR, and MCF-7/ADR cells were processed with 50/100/200/300/400/500/600 $\mu \mathrm{g} / \mathrm{L}$ ADR. Then the values of $\mathrm{IC}_{50}$ and cell viability in MCF-7/S and MCF-7/ADR cells were subsequently measured by MTT assay, while the apoptosis of MCF-7/S and MCF-7/ADR cells was 
A

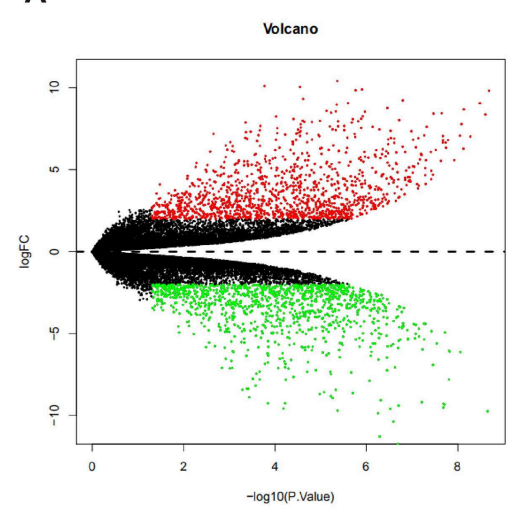

B

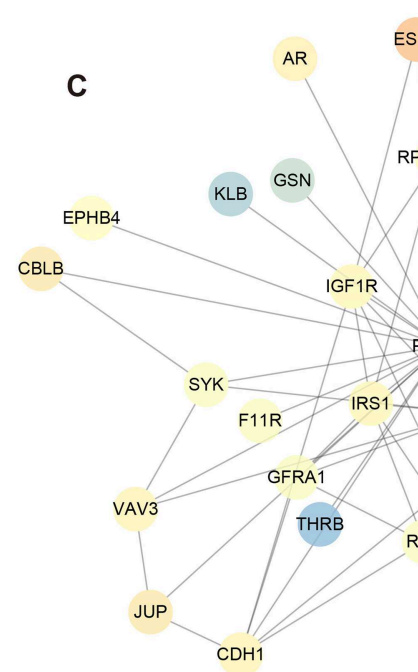

ESR 1
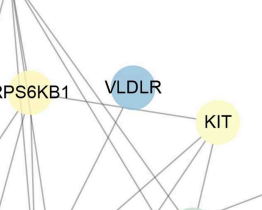

\section{NRG3

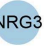

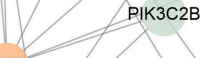
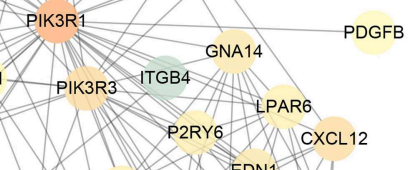

$\underbrace{\text { BDKRB2 }}_{\text {P2RY2 }}$
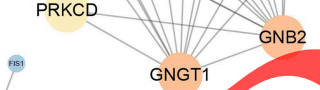
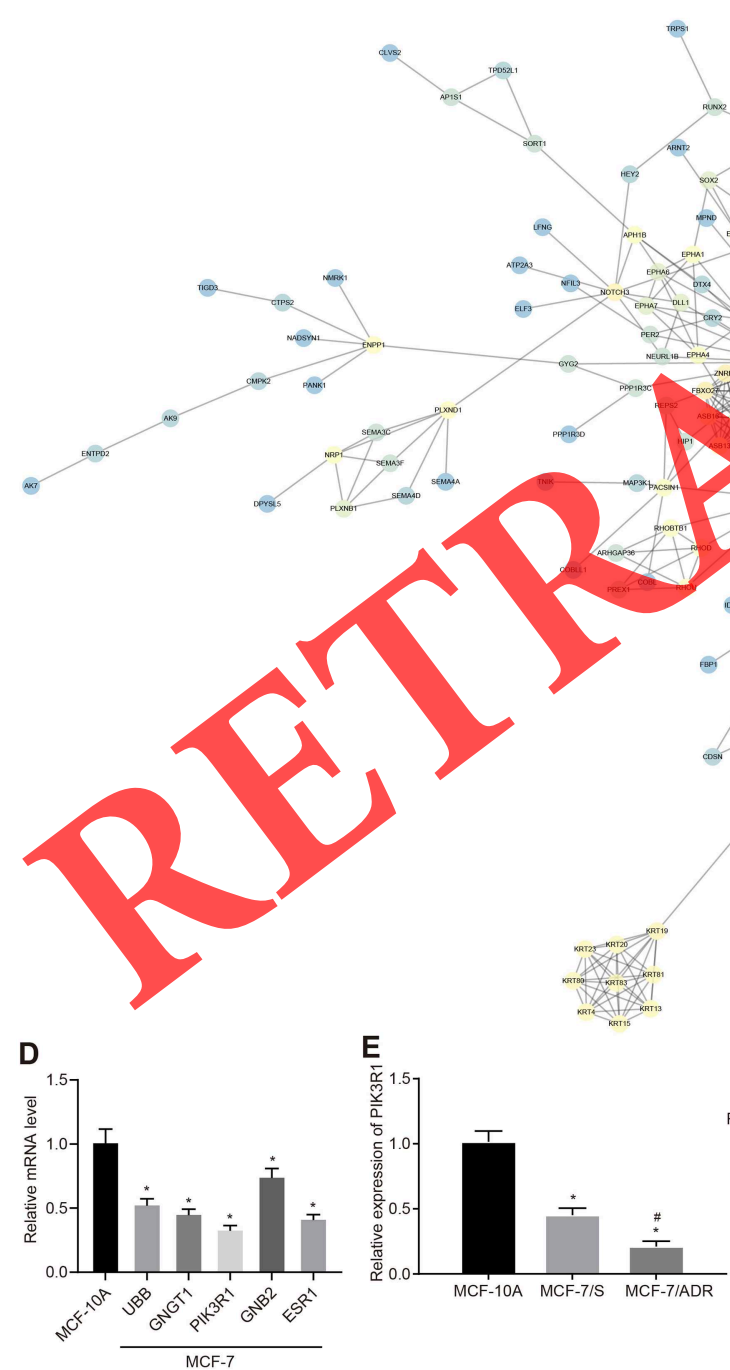

$\mathbf{F}$
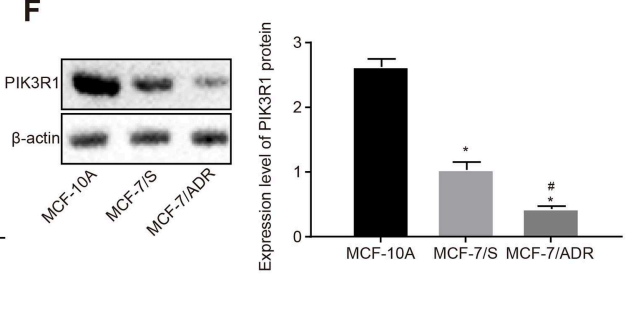

FIGURE 1 | PIK3R1 is poorly expressed in drug-resistant BC cells. (A) A volcano map depicting the expression of DEGs associated to the BC drug resistance in the GSE76540 dataset. $X$-axis indicated log10 $p$-value, and $Y$-axis represented logFC. Each point indicated a gene (red points represented upregulated DEGs in

(Continued) 
FIGURE 1 | drug-resistant samples and green points represented downregulated DEGs in drug-resistant samples). (B) PPI network of DEGs. Each circle indicated a gene, and the line between circles represented their interaction. The darker color indicated a higher core degree. (C) Sub-network of PIK3R1 interacting with PPI network. (D) Expression of UBB, GNGT1, PIK3R1, GNB2, and ESR1 in normal MCF-10A and ADR-sensitive MCF-7/S cells detected by RT-qPCR. (E) PIK3R1 expression in MCF-10A, MCF-7/S, and ADR-resistant MCF-7/ADR cells measured by RT-qPCR. (F) western blot analysis of PIK3R1 protein in MCF-10A, MCF-7/S, and MCF-7/ADR cells. Measurement data were expressed as mean \pm standard deviation. Data comparison among multiple groups was conducted by one-way ANOVA with Dunnett's post-hoc test. * $p<0.05$, compared with MCF-10A cells; \#p < 0.05, compared with MCF-7/S cells. Each experiment was repeated three times independently.

evaluated by flow cytometry. The results showed that PIK3R1 overexpression led to significantly augmented value of $\mathrm{IC}_{50}$ (Figures 2E,F), decreased cell viability (Figures 2G,H) and enhanced cell apoptosis (Figures 2I,J). However, the value of $\mathrm{IC}_{50}$ was notably diminished, while cell viability was elevated and cell apoptosis was declined in MCF-7/S and MCF-7/ADR cells when PIK3R1 was knocked down when compared with sh-NC treatment (all $p<0.05$ ). The aforementioned results provided evidence suggesting that PIK3R1 could affect drug resistance, cell viability, and apoptosis in BC cells.

\section{Isolation, Identification, and Endocytosis of MCF-7 Cell-Derived Exosomes}

As previously reported, BC-secreted exosomes participate in the drug resistance transfer $(6,18,19)$. Hence, we set out to elucidate the impact of exosomes contained in $\mathrm{BC}$ on cell drug resistance. Exosomes from MCF-7/S and MCF-7/ADR cells were isolated using high-speed centrifugation. TEM revealed that exosomes isolated from different cell lines had identical form (circular or oval membranous vesicle; Figure 3A). In addition, the diameter of the exosomal particles ranged from 30 to $120 \mathrm{~nm}$ in the determination of dynamic light scattering (Figure 3B). The expression of CD63 and HSP70 was then detected by western blot analysis, the results of which (Figure $3 \mathrm{C}$ ) demonstrated that CD63 and HSP70 were highly expressed in exosomes. The exosome surface marker CD63 content was subsequently identified by flow cytometry, which indicated that the CD63 content was markedly eleyated in exosomes isolated from cell lines $(p<0.05$; Figure 3D). The aforementioned results suggested that cellular exosomes were successfully isolated.

In order to verify, whether ADR-sensitive cells could endocytose MCF-7/ADR cell-derived exosomes, ADR exosomes traced by PKH67 (Green) were co-cultured with MCF-7/S cells for $48 \mathrm{~h}$. MCF-7/S endocytosing ADR exosomes were analyzed under a confocal microscopy. The results indicated notable MCF-7/S endocytosing ADR exosomes following $48 \mathrm{~h}$ of coculture (Figure 3E). The results clearly demonstrated that drugresistant exosomes could be endocytosed by ADR-sensitive cell line MCF-7/S.

\section{MCF-7/ADR Cell-Derived Exosomes Could Transfer Drug Resistance}

Next, to further investigate the role of exosomes in BC drug resistance transfer, exosomes secreted from different cells were processed with MCF-7/S cells and then treated with $500 \mathrm{ng} / \mathrm{mL}$ ADR for $48 \mathrm{~h}$. The value of $\mathrm{IC}_{50}$, cell viability and apoptosis in MCF-7/S cells were detected by MTT assay and flow cytometry, respectively. The results (Figures $4 \mathrm{~A}-\mathrm{C}$ ) exhibited that MCF7/S cells co-cultured with MCF-7/ADR cell-derived exosomes exhibited an elevated $\mathrm{IC}_{50}$-value and cell viability along with reduced cell apoptosis in contrast to MCF-7/S cells (all $p<0.05$ ); however, the MCF-7/S cells co-cultured with MCF-7/S cellderived exosomes displayed no significant difference $(p>0.05)$. These results demonstrated that exosomes in ADR-resistant cells could transfer drug resistance to ADR-sensitive cells, while ADR-sensitive cell-derived exosomes did not exert any influence on cell activity. Next, the expression of PIK3R1 was analyzed by RT-qPCR and western blot analysis. As illustrated in Figures 4D-E, protein level of PIK3R1 was decreased in MCF-7/ADR cell-derived exosomes $(p<0.05)$, but showed no significant change in MCF-7/S cell-derived exosomes $(p>0.05)$, when compared to the MCF- $7 / S$ cells. Taken together, MCF7/ADR cell-derived exosomes could inhibit PIK3R1 to regulate cell viability, apoptosis and drug resistance.

\section{PIK3R1 Was a Target Gene of miR-221-3p}

In an attempt to clarify the upstream regulatory mechanism of drug-resistant BC cell-derived exosomes affecting BC drug resistance, upregulated miRNAs differentially expressed in $\mathrm{BC}$ exosomes were obtained from the EVmiRNA database. Next, based on the mirDIP and TargetScan databases, the upstream regulatory miRNA of PIK3R1 was predicted, which was jointly analyzed via the EVmiRNA database (Figure 5A). There were 5 miRNAs emerged from the above analyses (hsa-miR-2213p, hsa-miR-320a, hsa-miR-93-5p, hsa-miR-21-5p, and hsa-miR222-3p), among which miR-221-3p displayed the most distinct altered expression (Figure 5B). A specific binding region was detected between the sequence of PIK3R1 and miR-221-3p based on the analysis conducted by the online software (Figure 5C).

The expression of miR-221-3p was detected by RT-qPCR in exosomes of drug-sensitive cells and drug-resistant cells. Furthermore, miR-221-3p exhibited higher expression in the exosomes of ADR-resistant cells than that in exosomes of drugsensitive cells $(p<0.05)$ (Figure 5D). The targeting relationship between miR-221-3p and PIK3R1 was subsequently verified using dual-luciferase reporter assay. The results shown in Figure 5E presented that the luciferase activity of wild type (WT)-PIK3R1 was reduced in cells upon miR-221-3p mimic treatment in contrast to mimic NC treatment $(p<0.05)$ while the luciferase activity of mutant (Mut)-PIK3R1 showed no significant change $(p>0.05)$. These results indicated that miR-221-3p could specifically bind to PIK3R1. The altered expression of miR-221-3p in MCF-7/ADR cells was further identified, and meanwhile, PIK3R1 expression in the MCF-7/ADR cells was analyzed by RT-qPCR and western blot analysis (Figures 5F,G). 


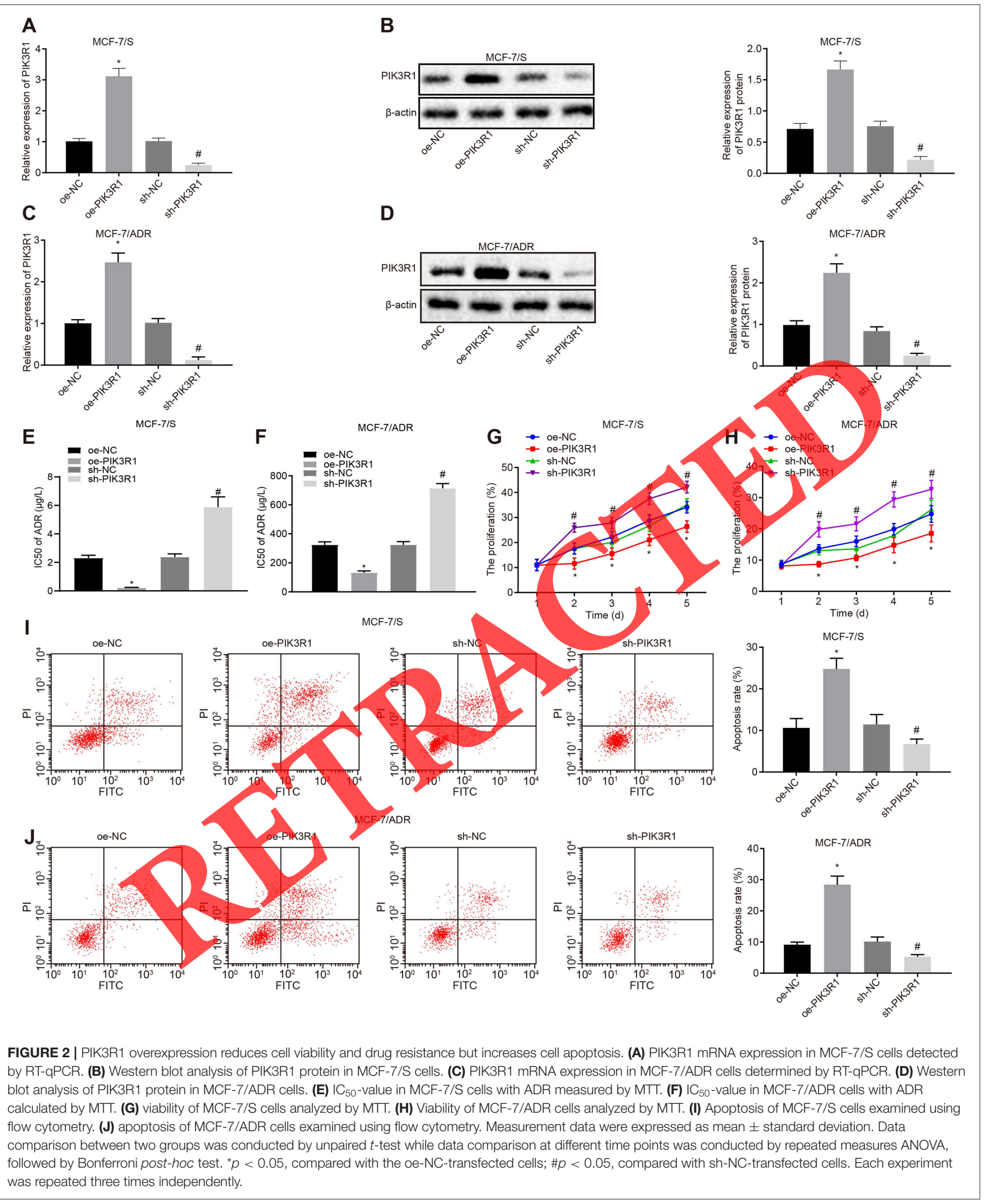




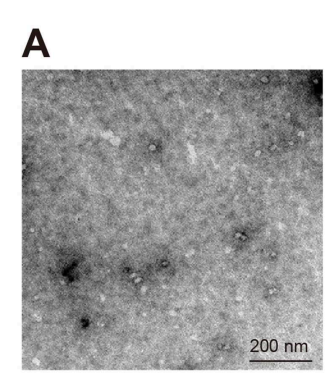

E

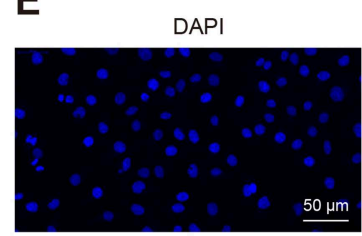

B

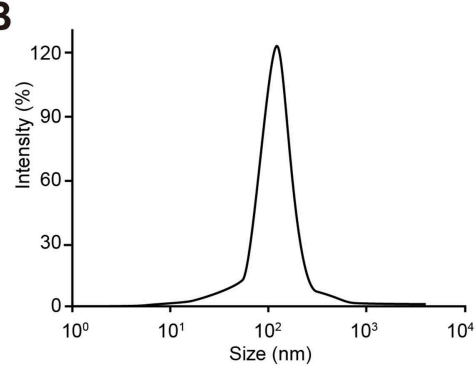

PKH67-labeled MSCs-Exo

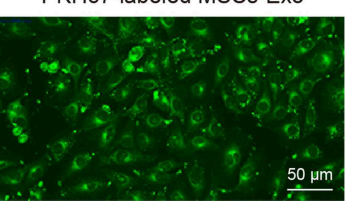

C
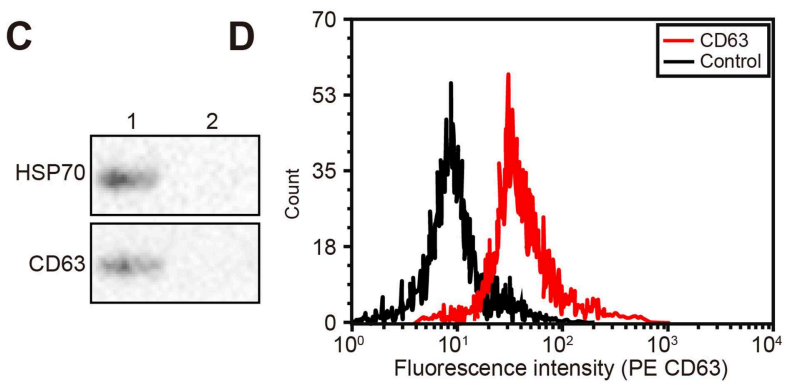

FIGURE 3 | MCF-7/S cells endocytose MCF-7/ADR cell-derived exosomes. (A) Exosome identified by a TEM (200 nm). (B) Diameter of exosomes measured by dynamic light scattering. (C) Western blot analysis of exosomal surface markers CD63 and HSP70 proteins. Lane 1: isolated exosome; lane 2: supernatant of isolated exosome. (D) CD63 content determined by flow cytometry. (E) Glioma cell endocytosing exosome analyzed under an inverted fluorescence microscope (200x). The measurement data were expressed as mean \pm standard deviation. Data comparison between two groups was conducted by unpaired $t$-test. Each experiment was repeated three times independently.

Compared with cells transfected with mimic NC, PIK3R1 mRNA and protein level were notably decreased in cells transfected with miR-221-3p mimic, while they were obviously elevated in cells transfected with miR-221-3p inhibitor in contrast to inhibitor NC-transfected cells (all $p<0.05$ ). The above results indicated that PIK3R1 was regulated by miR-221-3p. Upregulated miR221-3p could inhibit PIK3R1 expression, while downregulated miR-221-3p promoted PIK3R1 expression.

\section{Exosomal miR-221-3p Promoted BC Cell Drug Resistance by Targeting PIK3R1 and Inhibiting the PI3K/AKT Signaling Pathway} We next aimed to further investigate whether drug-resistant exosomes promoted drug resistance via miR-221-3p or not, MCF-7/ADR cells were transfected with miR-221-3p mimic or inhibitor. Then, MCF-7/S cells were processed with isolated exosomes. miR-221-3p expression and PIK3R1 mRNA expression were detected by RT-qPCR, while PIK3R1 protein level was determined by western blot analysis. As illustrated in Figures 6A,B, when MCF-7/ADR cell-derived exosomes were co-cultured with miR-221-3p mimic, miR-221-3p was upregulated but PIK3R1 was downregulated, while a contrasting trend was identified in the expression of miR-221-3p and PIK3R1 in MCF-7/ADR cell-derived exosomes co-cultured with miR-221-3p inhibitor. After ADR treatment, the value of $\mathrm{IC}_{50}$ was measured in MCF-7/S cells by MTT assay. The $\mathrm{IC}_{50}$-value was increased in MCF-7/ADR cell-derived exosomes treated with miR-221-3p mimic, but it was decreased in MCF7/ADR cell-derived exosomes treated with miR-221-3p inhibitor (Figure 6C). MTT assay and flow cytometry (Figures 6D,E) revealed that cell viability was increased but cell apoptosis was decreased in MCF-7/ADR cell-derived exosomes treated with miR-221-3p mimic, all of which was reversed in MCF-7/ADR cell-derived exosomes treated with miR-221-3p inhibitor (all $p$ $<0.05)$. These results revealed that miR-221-3p contained in exosomes promoted drug resistance of BC cells. As previously suggested in literature, PIK3R1 regulated drug resistance through the PI3K/AKT signaling pathway (20). In order to validate this, western blot analysis was performed to detect the extent of PI3K and AKT phosphorylation as well as protein expression of PI3K and $A K T$. The results shown in Figure 6F displayed an upward trend in the protein expression of PI3K and AKT as well as the extent of PI3K and AKT phosphorylation in MCF-7/ADR cell-derived exosomes treated with miR-221-3p mimic, which was abrogated in MCF-7/ADR cell-derived exosomes treated with miR-221-3p inhibitor (all $p<0.05$ ). All the results indicated that exosomes containing miR-221-3p targeted PIK3R1 and affected drug resistance via the PI3K/AKT signaling pathway.

\section{Inhibited Exosomal miR-221-3p Derived From MCF-7/ADR Cells Suppressed Tumor Formation in vivo}

Lastly, we attempted to study the impact of MCF-7/ADR exosomal miR-221-3p on BC tumor formation in nude mice. Xenograft tumor model was first induced in nude mice. After tumor formation, the mice were then subjected to different treatments. Tumor growth curve, volume, and weight were then analyzed. The results shown in Figures 7A-D presented that tumor growth speed, volume, and weight of mice were significantly reduced in MCF-7/ADR cell-derived exosomes treated with miR-221-3p inhibitor and ADR, when compared with those in MCF-7/ADR cell-derived exosomes treated with inhibitor NC and ADR $(p<0.05)$.

Cell apoptosis was determined using flow cytometry, and protein expression of PI3K and AKT as well as the extent of $\mathrm{PI} 3 \mathrm{~K}$ and $\mathrm{AKT}$ phosphorylation was evaluated by western blot 


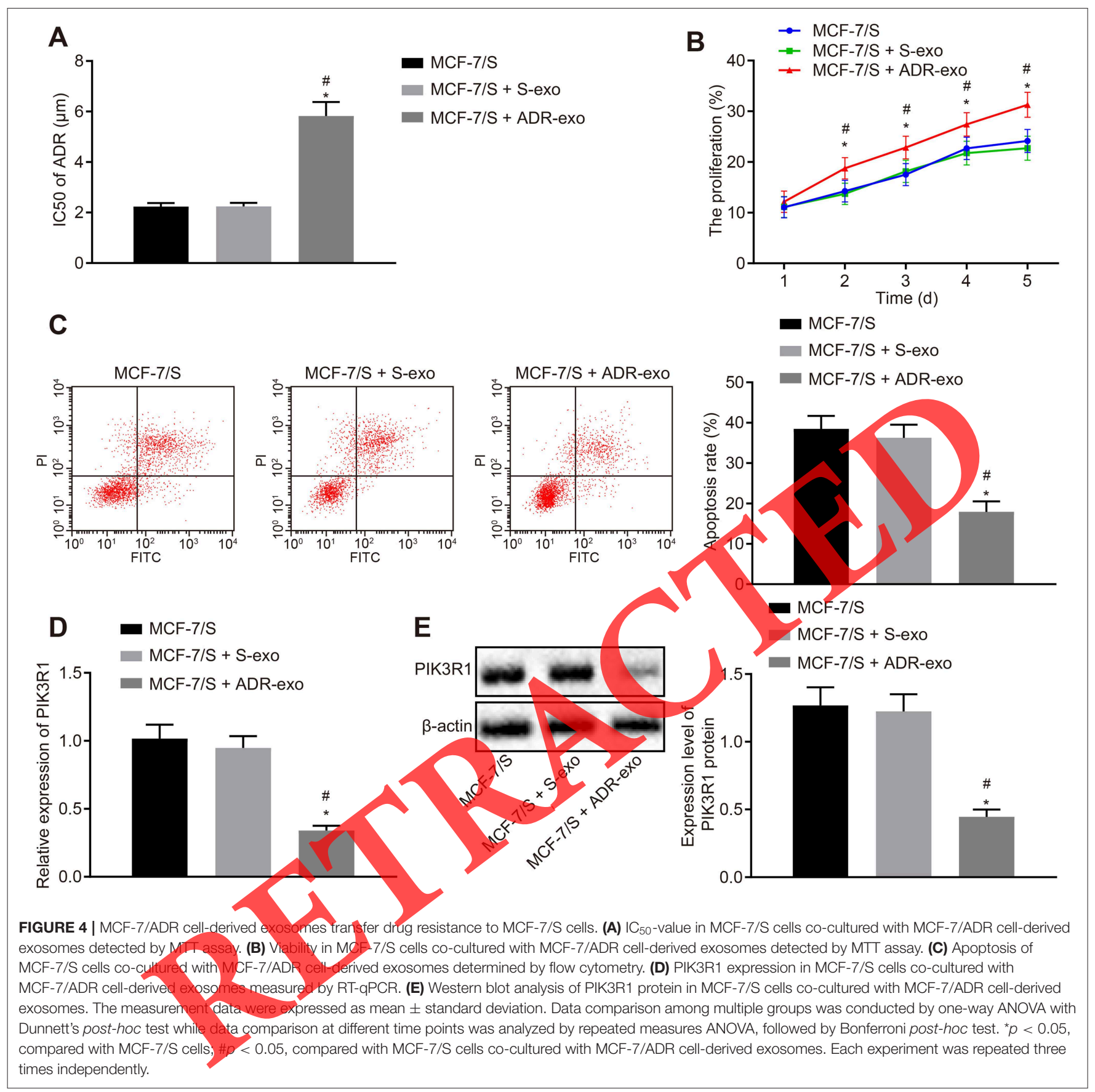

analysis. Results exhibited that cell apoptosis was decreased in MCF-7/ADR cells following ADR treatment in contrast to that following PBS treatment. Cell apoptosis, along with protein expression of PI3K and AKT as well as the extent of PI3K and AKT phosphorylation was reduced in MCF-7/ADR cell-derived exosomes treated with both miR-221-3p inhibitor and ADR in contrast to those in MCF-7/ADR cell-derived exosomes treated with inhibitor NC and ADR (all $p<0.05$; Figures 7E,F). These findings further proved that inhibition of exosomal miR-221-3p derived from MCF-7/ADR cells and ADR could retard tumor formation in nude mice.

\section{DISCUSSION}

$\mathrm{BC}$, the second major cause of cancer deaths in women, affects $\sim 1.7$ million individuals every year worldwide and the drug resistance remains an obstacle of developing more effective and efficient therapeutics (5). Exosomal miRNAs possess the ability to shuttle between cells to communicate and exchange genetic material valuable, highlighting them as candidate biomarkers for tumorigenesis and drug resistance (21), particularly in BC (22). The gathered findings from the present study demonstrated that exosomal miR-221-3p could enhance the resistance of 

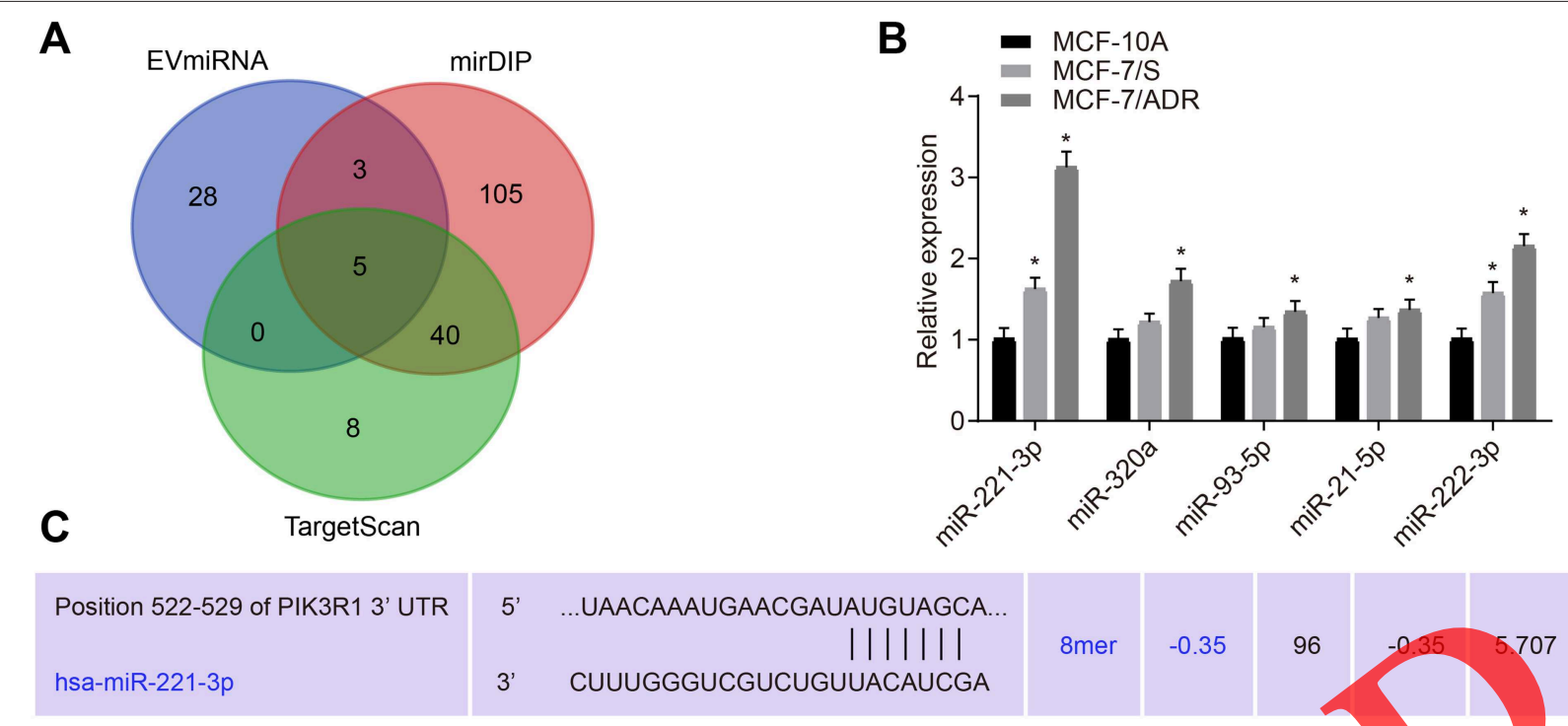

D

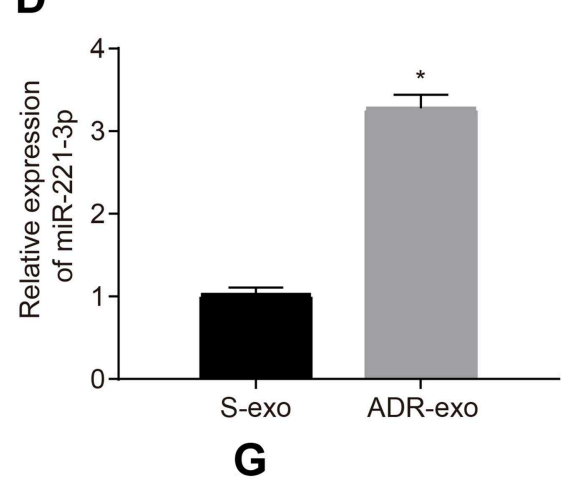

\section{$\mathbf{E}$}
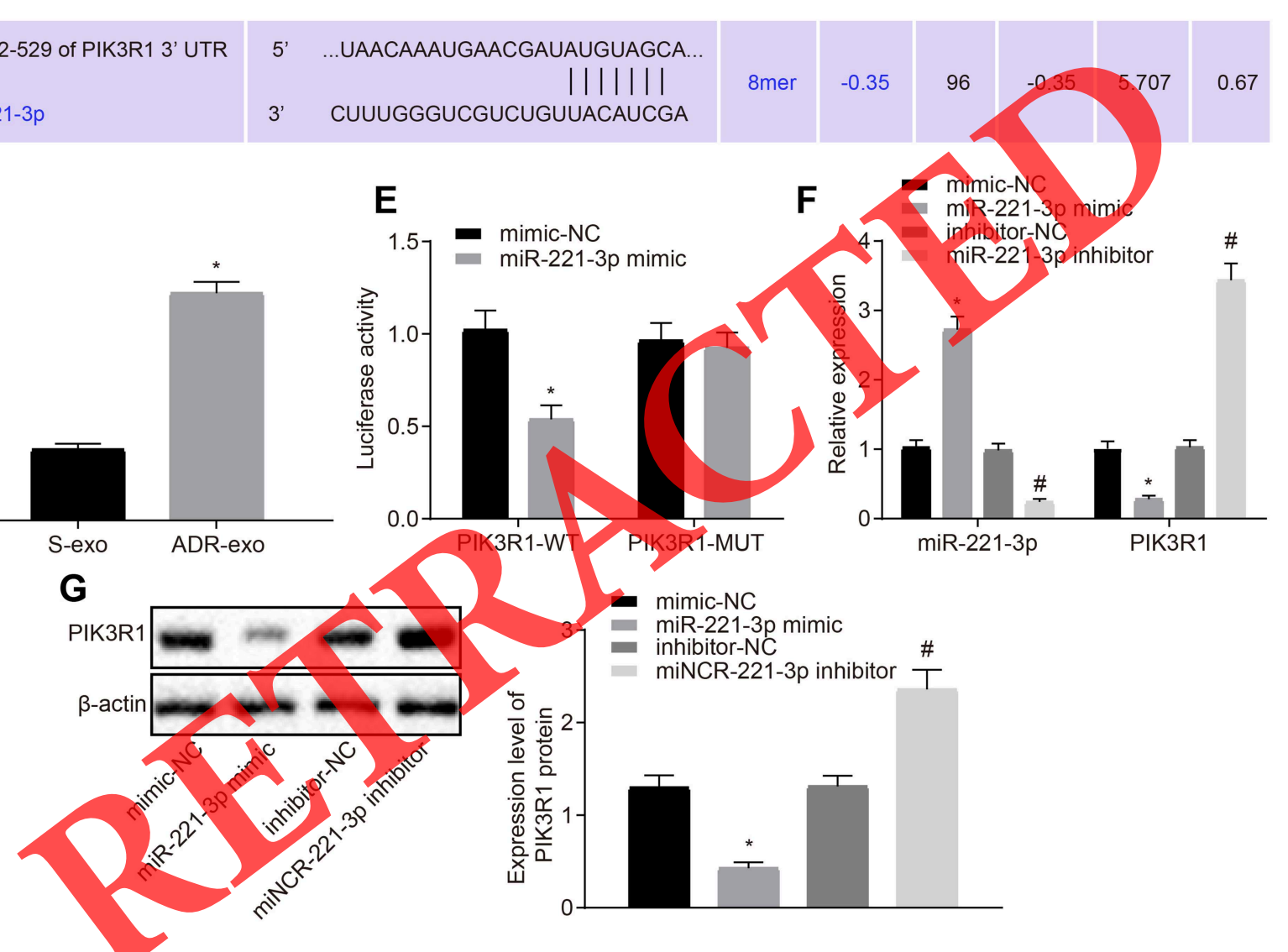

FIGURE 5 | BC cell exosomal miR-221-3p targets PIK3R1. (A) Upstream regulatory miRNAs of PIK3R1 predicted using the mirDIP and TargetScan databases. Three circles were indicative of the results obtained from the EVmiRNA, mirDIP, and TargetScan databases, respectively, and the middle part denoted the intersected results of these three databases. (B) miRNA expression in MCF-10A, MCF-7/S, and MCF-7/ADR cells detected by RT-qPCR. * $p<0.05$ against MCF-10A cells. (C) Binding sites between PIK3R1 and miR-221-3p analyzed using an online software. (D) miR-221-3p expression in exosomes derived from ADR-sensitive and ADR-resistant cells analyzed by RT-qPCR. (E) The binding of PIK3R1 to miR-221-3p verified by dual-luciferase reporter assay. (F) PIK3R1 expression in MCF-7/ADR cells transfected with miR-221-3p mimic or inhibitor measured by RT-qPCR. (G) Western blot analysis of PIK3R1 protein in MCF-7/ADR cells transfected with miR-221-3p mimic or inhibitor. The measurement data were expressed as mean \pm standard deviation. Data comparison between two groups was conducted by unpaired $t$-test, while data comparison among multiple groups was conducted by one-way ANOVA with Dunnett's post-hoc test. ${ }^{*} p<0.05$, compared with mimic NC-transfected cells; $\# p<0.05$, compared with inhibitor NC-transfected cells. Each experiment was repeated three times independently.

BC cells to ADR by targeting PIK3R1 via the PI3K/AKT signaling pathway.

During the current study, the MCF-7/ADR cell line exhibited high levels of miR-221-3p expression. In a recent study,
miR-221-3p was reported to be up-regulated in both resistant BC tissues and cells (23). More recently, the exosome-mediated transfer of miRNAs has been delineated to alter BC cell resistance to drugs, unearthing potential biomarkers for $\mathrm{BC}$ progression 


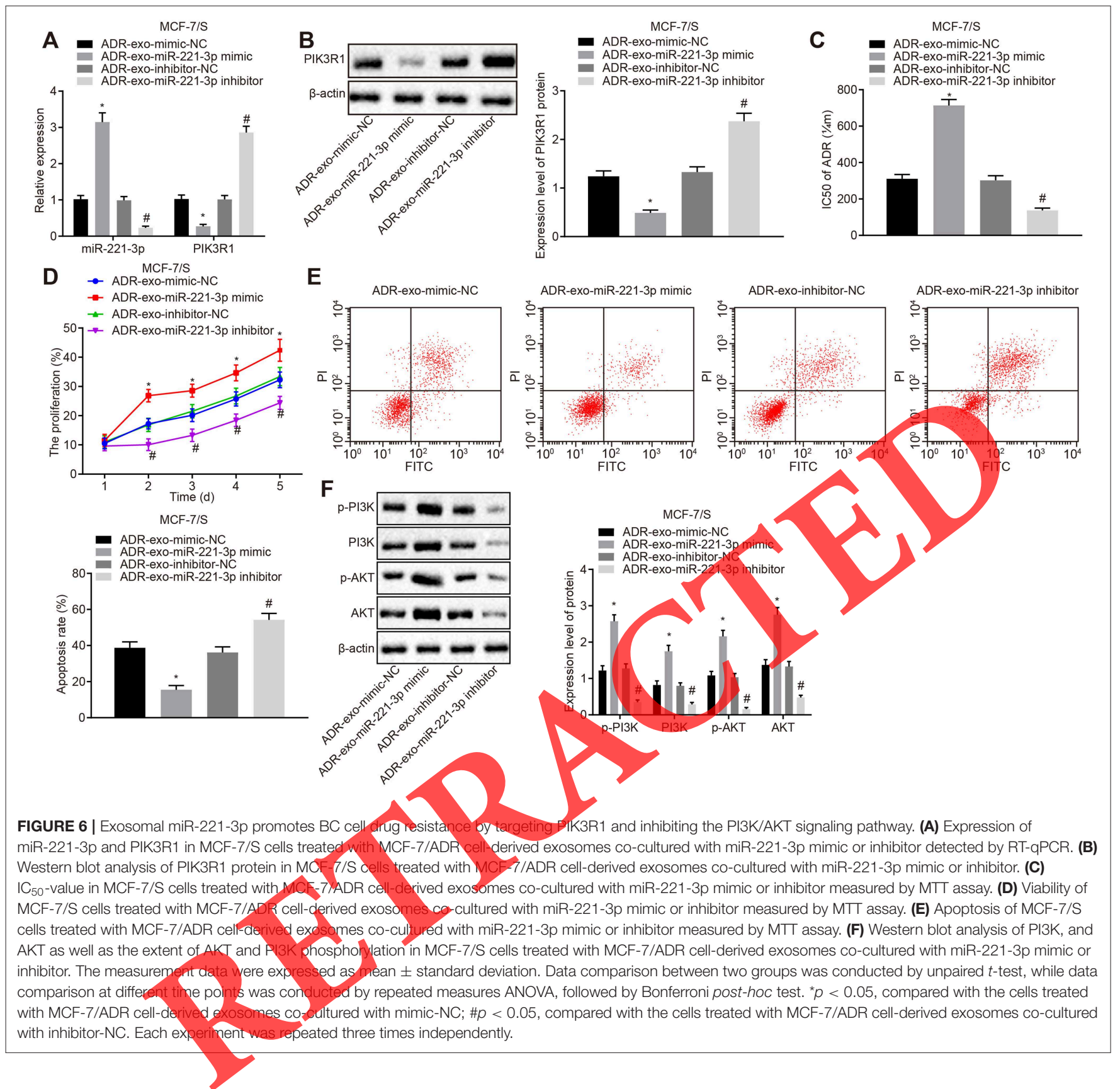

and treatment (24). Exosomal miR-221 exerts promoting effects on the resistance of glioma cells to temozolomide, thus inducing tumor progression (25). In pancreatic cancer, miR-221-3p has also been reposted to be responsible for the drug resistance (26). These findings are partially in agreement with ours, whereby exosomal miR-221-3p could promote the resistance of BC cells to ADR. Furthermore, the expression of hsa-miR-221-3p may be associated with the diagnosis and prognosis of $\mathrm{BC}$ patients (27).

The downstream regulatory mechanism of miR-221-3p was subsequently analyzed. PIK3R1 was proven to be a target gene of miR-221-3p. Through analysis of BC drug resistance-related microarray data, PIK3R1 was identified. PIK3R1 expression was decreased both at mRNA level and protein level in MCF-7/ADR cells. PIK3R1 is defined as a differentially expressed gene in invasive BC (28), which was consistent with our research. In addition, PIK3R1 is also found to be expressed at a poor level in BC cells (29). In a previous study, PIK3R1 is linked to insulin resistance in vivo (30). Moreover, PIK3R1 is regarded as one of the biomarkers predicting drug resistance in human epidermal growth factor receptor-2 $2^{+}$cancer cells (31). Also, CapG-mediated hyperactivation of PIK3R1 results in increased sensitivity to chemotherapy in BC cells (16). The role of PIK3R1 in BC was further analyzed, which revealed the role of $\mathrm{PIK} 3 \mathrm{R} 1$ in the invasion and migration of MCF-7 cells (32), confirming that PIK3R1 is associated 


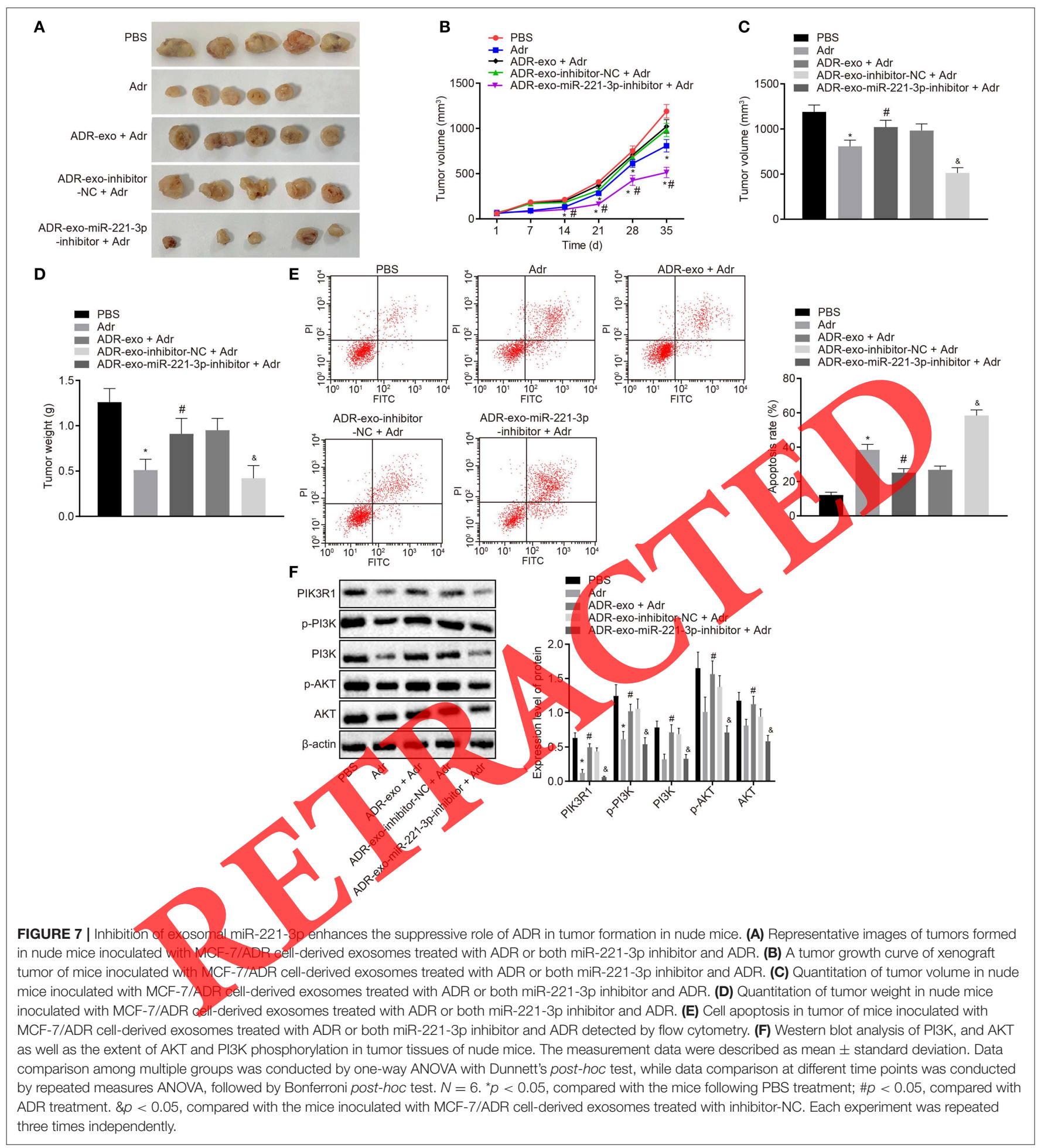

with human BC epithelial cell line. However, there are few studies investigating the altered expression of PIK3R1 in BC and the relationship between PIK3R1 and BC drug resistance, while the current study conducted an in-depth investigation into this topic. The influence of PIK3R1 in the event of $\mathrm{BC}$ drug resistance was determined using ectopic expression experiments and the results in our study proved that the overexpressed PIK3R1 regulated drug resistance and resulted in an increase in cell apoptosis and diminished cell viability in BC. PIK3R1 has been reported to be negatively regulated by miR-21 in BC (20). Besides, the binding between PIK3R1 and miR-221 had been previously reported, with miR-221 


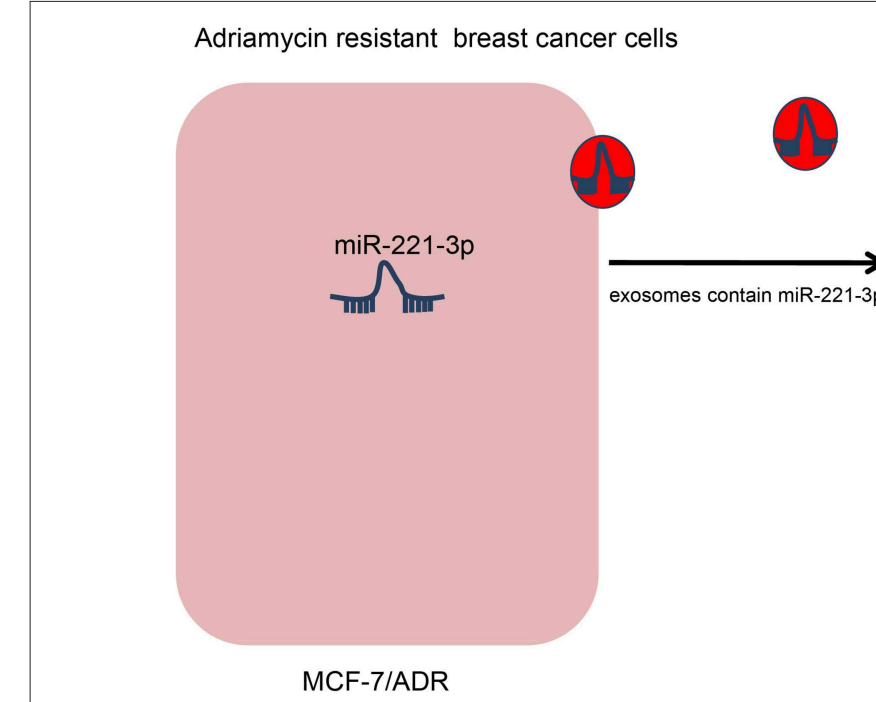

Adriamycin sensitive breast cancer cells

FIGURE 8 | The graph of the molecular mechanism of miR-221-3p in ADR-resistant BC cells. In ADR-resistant BC cells negatively regulating PIK3R1, thereby promoting $B C$ cell viability and inhibiting apoptosis. In addition, miR-221-3p ca ADR-resistant BC cells, and transferred to ADR-sensitive BC cells.

found to target PIK3R1 in endothelial progenitor cells (33). During the current study, bioinformatics analysis and dualluciferase reporter assay confirmed that exosomal miR-221$3 p$ could target PIK3R1 and inhibit its expression in BCresistant cells.

Based on the known regulatory mechanism underlying miR-221-3p/PIK3R1, our data further verified the mechanism by which exosomal miR-221-3p/PIK3R1 influences BC drug resistance. Our results indicated that exosomal inhibited the PI3K/AKT signaling pathway by targeting PIK3R1. Activation of the PI3K/AKT signaling pathway is customarily regarded as an emblem of $\mathrm{BC}$ developnent (33). Furthermore, the mutant PI3K/AKT/mTOR pathway is frequenty found in BC drug resistance (34). Consistent with the findings of the current study, inhibition of the PI3K/AKT signaling pathway has been reported to induce drug resistance in gastric cancer cells (35). As highlighted in our study, exosomal miR-221-3p-mediated PIK3R1 downregulation promoted BC drug resistance by suppressing the PI3K/AKT signaling pathway. The in vivo experimental results further confirmed that the regulatory network contributed to tumor formation.

In conclusion, drug-resistant $\mathrm{BC}$ cells-derived exosomal miR-221-3p could promote drug resistance of BC cells to ADR by targeting PIK3R1 via PI3K/AKT signaling pathway in vitro and in vivo (Figure 8). These findings may provide further perspective and insight for future investigations into the mechanism of BC drug resistance. However, this research is still at a preliminary stage, further investigation is still required regarding the action of the mechanism.

\section{DATA AVAILABILITY STATEMENT}

Publicly available datasets were analyzed in this study, these can be found in the NCBI Gene Expression Omnibus (GSE76540).

\section{ETHICS STATEMENT}

This study was approved by the Animal Ethics Committee of Huadu Hospital, Southern Medical University. Nude mice were employed in this study according to the Guidelines for the Care and Use of Laboratory Animals published by the National Institutes of Health.

\section{AUTHOR CONTRIBUTIONS}

$\mathrm{XP}, \mathrm{XH}$, and RW wrote the paper and conceived and designed the experiments. JL, NH, and LC analyzed the data. YC and MY collected and provided the sample for this study. All authors have read and approved the final submitted manuscript.

\section{FUNDING}

This work was supported by Guangzhou Health Science and Technology Project (No. 20191A011115) and the Scientific Research Fund of Huadu Hospital, Southern Medical University (No. 2019A04).

\section{ACKNOWLEDGMENTS}

We would like to express our sincere appreciation to the reviewers for critical comments on this article. 


\section{REFERENCES}

1. Phung MT, Tin S, Elwood JM. Prognostic models for breast cancer: a systematic review. BMC Cancer. (2019) 19:230. doi: 10.1186/s12885-019-5442-6

2. Wang K, Ren Y, Li H, Zheng K, Jiang J, Zou T, et al. Comparison of clinicopathological features and treatments between young $(</=40$ years $)$ and older ( $>40$ years) female breast cancer patients in West China: a retrospective, epidemiological, multicenter, case only study. PLoS One. (2016) 11:e0152312. doi: 10.1371/journal.pone.0152312

3. Huang J, Li H, Ren G. Epithelial-mesenchymal transition and drug resistance in breast cancer (Review). Int J Oncol. (2015) 47:840-8. doi: 10.3892/ijo.2015.3084

4. Gottesman MM, Lavi O, Hall MD, Gillet JP. Toward a better understanding of the complexity of cancer drug resistance. Annu Rev Pharmacol Toxicol. (2016) 56:85-102. doi: 10.1146/annurev-pharmtox-010715-103111

5. Tang Y, Wang Y, Kiani MF, Wang B. Classification, treatment strategy, and associated drug resistance in breast cancer. Clin Breast Cancer. (2016) 16:33543. doi: 10.1016/j.clbc.2016.05.012

6. Wang X, Xu C, Hua Y, Sun L, Cheng K, Jia Z, et al. Exosomes play an important role in the process of psoralen reverse multidrug resistance of breast cancer. J Exp Clin Cancer Res. (2016) 35:186. doi: 10.1186/s13046-016-0468-y

7. Yu DD, Wu Y, Shen HY, Lv MM, Chen WX, Zhang XH, et al. Exosomes in development, metastasis and drug resistance of breast cancer. Cancer Sci. (2015) 106:959-64. doi: 10.1111/cas.12715

8. Najminejad H, Kalantar SM, Abdollahpour-Alitappeh M, Karimi MH, Seifalian AM, Gholipourmalekabadi M, et al. Emerging roles of exosomal miRNAs in breast cancer drug resistance. IUBMB Life. (2019) 71:1672-84. doi: 10.1002/iub.2116

9. Wu XG, Zhou CF, Zhang YM, Yan RM, Wei WF, Chen XJ, et al. Cancerderived exosomal miR-221-3p promotes angiogenesis by targeting THBS2 in cervical squamous cell carcinoma. Angiogenesis. (2019) 22:397-410. doi: 10.1007/s10456-019-09665-1

10. Acunzo M, Visone R, Romano G, Veronese A, Lovat F, Palmieri $\mathrm{D}$, et al. miR-130a targets MET and induces TRAIL-sensitivity in NSCLC by downregulating miR-221 and 222. Oncogene. (2012) 31:634-42. doi: 10.1038/onc.2011.260

11. Mandujano-Tinoco EA, Garcia-Venzor A, Munoz-Galindo L, LizarragaSanchez F, Favela-Orozco A, Chavez-Gutierrez E, et al. miRNA expression profile in multicellular breast cancer spheroids. Biochim Biophys Acta Mol Cell Res. (2017) 1864:1642-55. doi: 10.1016/j.bbamcr.2017.05.023

12. Ergun S, Tayeb TS, Arslan A, Temiz E, Arman K, Safdar M, et al. The investigation of miR-221-3p and PAK1 gene expressions in breast cancer cell lines. Gene. (2015) 555:377-81. doi. 10.1016/j.gene.2014.11.036

13. Fang R, Zhu Y, Hu L, Khadka XS, Á̉, Zou H, et al. Plasma microRNA pair panels as novel biomarkers for detection of early stage breast cancer. Front Physiol. (2018) 9:1879. doi: 10.3389/fphys.2018.01879

14. Mavrogiannis AV, Kokkinopoulou I, Kontos CK, Sideris DC. Effect of vinca alkaloids on the expression levels of microRNAs targeting apoptosis-related genes in breast cancer cell lines. Curr Pharm Biotechnol. (2018) 19:1076-86. doi: $10.2174 / 1389201019666181112103204$

15. Okamoto K, Miyoshi K, Murawaki Y. miR-29b, miR-205 and miR-221 enhance chemosensitivity to gemcitabine in $\mathrm{HuH} 28$ human cholangiocarcinoma cells. PLoS One. (2013) 8:e77623. doi: 10.1371/journal.pone.0077623

16. Chi Y, Xue J, Huang S, Xiu B, Su Y, Wang W, et al. CapG promotes resistance to paclitaxel in breast cancer through transactivation of PIK3R1/P50. Theranostics. (2019) 9:6840-55. doi: 10.7150/thno.36338

17. Zhang G, Zou X, Miao S, Chen J, Du T, Zhong L, et al. The anti-oxidative role of micro-vesicles derived from human Wharton-Jelly mesenchymal stromal cells through NOX2/gp91(phox) suppression in alleviating renal ischemia-reperfusion injury in rats. PLoS One. (2014) 9:e92129. doi: 10.1371/journal.pone.0092129

18. Ning K, Wang T, Sun X, Zhang P, Chen Y, Jin J, et al. UCH-L1-containing exosomes mediate chemotherapeutic resistance transfer in breast cancer. $J$ Surg Oncol. (2017) 115:932-40. doi: 10.1002/jso.24614

19. Guo J, Hao J, Jiang H, Jin J, Wu H, Jin Z, et al. Proteasome activator subunit 3 promotes pancreatic cancer growth via c-Myc-glycolysis signaling axis. Cancer Lett. (2017) 386:161-7. doi: 10.1016/j.canlet.2016.08.018
20. Yan LX, Liu YH, Xiang JW, Wu QN, Xu LB, Luo XL, et al. PIK3R1 targeting by miR-21 suppresses tumor cell migration and invasion by reducing PI3K/AKT signaling and reversing EMT, and predicts clinical outcome of breast cancer. Int J Oncol. (2016) 48:471-84. doi: 10.3892/ijo.2015. 3287

21. Zhao L, Liu W, Xiao J, Cao B. The role of exosomes and "exosomal shuttle microRNA" in tumorigenesis and drug resistance. Cancer Lett. (2015) 356:339-46. doi: 10.1016/j.canlet.2014.10.027

22. Sempere LF, Keto J, Fabbri M. Exosomal microRNAs in breast cancer towards diagnostic and therapeutic applications. Cancers (Basel). (2017) 9:E71. doi: 10.3390/cancers9070071

23. Chen Z, Pan T, Jiang D, Jin L, Geng Y, Feng X, et al. The lncRNA-GAS5/miR221-3p/DKK2 axis modulates the ABCB1-mediated adriamycin resistance of breast cancer via Wnt/ $\beta$-catenin signal pathway. Mol Ther Nucleic Acids. (2020) 19:1434-48. doi: 10.1016/j.omtn.2020.01.030

24. Santos JC, Ribeiro ML, Sarian LO, Ortega MM, Derchain SF. Exosomesmediate microRNAs transfer in breast cancer chemoresistance regulation. Am J Cancer Res. (2016) 6:2129-39.

25. Yang JK, Yang JP, Tong J, Jing SY, Fan B, Wang F, et al. Exosomal miR-221 targets DNM3 to induce tumor progression and temozolomide resistance in glioma. J Neurooncol. (2017) 131:255-65. doi: 10.1007/s11060-0162308-5

26. Zhao L, Zou D, Wei X, Wang L, Zhang Y, Liu S, et al, MiRNA-221-3p desensitizes pancreatic cancer cells to 5 -fluorouracil by targeting RB1. Tumour Biol. (2016) 37:16053-63. doi: 10.1007/s13277-016-5445-8

27. Abak A, Amini S, Estian MA, MontazeriV, Sakhinia E, Abhari A. Analysis of miRNA-221 expression level in tumors and marginal biopsies from patients with breast cancer (cross-sectional observational study). Clin Lab. (2018) 64:169-75. doi: 10.7754/Clin.Lab.2017.170821

28. Zhang BH, Liu J, Zhou QX, Zuo D, Wang Y. Analysis of differentially expressed genes in ductal carcinoma with DNA microarray. Eur Rev Med Pharmacol Sci. (2013) 17:758-66.

29. Kim S, Lee E, Jung J, Lee JW, Kim HJ, Kim J, et al. microRNA-155 positively regulates glucose metabolism via PIK3R1-FOXO3a-cMYC axis in breast cancer. Oncogene. (2018) 37:2982-91. doi: 10.1038/s41388-018-0124-4

Winnay JN, Solheim MH, Dirice E, Sakaguchi M, Noh HL, Kang HJ, et al. PI3kinase mutation linked to insulin and growth factor resistance in vivo. J Clin Invest. (2016) 126:1401-12. doi: 10.1172/JCI84005

Kirouac DC, Du J, Lahdenranta J, Onsum MD, Nielsen UB, Schoeberl B, et al. HER2 + cancer cell dependence on PI3K vs. MAPK signaling axes is determined by expression of EGFR, ERBB3 and CDKN1B. PLoS Comput Biol. (2016) 12:e1004827. doi: 10.1371/journal.pcbi.1004827

32. Uchino M, Kojima H, Wada K, Imada M, Onoda F, Satofuka H, et al. Nuclear beta-catenin and $\mathrm{CD} 44$ upregulation characterize invasive cell populations in non-aggressive MCF-7 breast cancer cells. BMC Cancer. (2010) 10:414. doi: 10.1186/1471-2407-10-414

33. Chang TY, Huang TS, Wang HW, Chang SJ, Lo HH, Chiu YL, et al. miRNome traits analysis on endothelial lineage cells discloses biomarker potential circulating microRNAs which affect progenitor activities. BMC Genomics. (2014) 15:802. doi: 10.1186/1471-2164-15-802

34. Guerrero-Zotano A, Mayer IA, Arteaga CL. PI3K/AKT/mTOR: role in breast cancer progression, drug resistance, and treatment. Cancer Metastasis Rev. (2016) 35:515-24. doi: 10.1007/s10555-016-9637-x

35. Liang J, Ge F, Guo C, Luo G, Wang X, Han G, et al. Inhibition of PI3K/Akt partially leads to the inhibition of $\operatorname{PrP}(\mathrm{C})$-induced drug resistance in gastric cancer cells. FEBS J. (2009) 276:685-94. doi: $10.1111 /$ j.1742-4658.2008.06816.x

Conflict of Interest: The authors declare that the research was conducted in the absence of any commercial or financial relationships that could be construed as a potential conflict of interest.

Copyright (c) 2020 Pan, Hong, Lai, Cheng, Cheng, Yao, Wang and Hu. This is an open-access article distributed under the terms of the Creative Commons Attribution License (CC BY). The use, distribution or reproduction in other forums is permitted, provided the original author(s) and the copyright owner(s) are credited and that the original publication in this journal is cited, in accordance with accepted academic practice. No use, distribution or reproduction is permitted which does not comply with these terms. 